\title{
A Primer on Large Intelligent Surface (LIS) for Wireless Sensing in an Industrial Setting ${ }^{\star}$
}

\author{
Cristian J. Vaca-Rubio ${ }^{1}$, Pablo Ramirez-Espinosa ${ }^{1}$, Robin Jess Williams ${ }^{1}$, \\ Kimmo Kansanen ${ }^{2}$, Zheng-Hua Tan ${ }^{1}$, Elisabeth de Carvalho ${ }^{1}$, and Petar \\ Popovski $^{1}$ \\ 1 Department of Electronic Systems, Aalborg University, Denmark \\ $\{c j v r$, pres, rjw, zt, edc, petarp\}@es.aau.dk \\ 2 Norwegian University of Science and Technology, Trondheim, Norway \\ kimmo.kansanen@ntnu.no
}

\begin{abstract}
One of the beyond-5G developments that is often highlighted is the integration of wireless communication and radio sensing. This paper addresses the potential of communication-sensing integration of Large Intelligent Surfaces (LIS) in an exemplary Industry 4.0 scenario. Besides the potential for high throughput and efficient multiplexing of wireless links, an LIS can offer a high-resolution rendering of the propagation environment. This is because, in an indoor setting, it can be placed in proximity to the sensed phenomena, while the high resolution is offered by densely spaced tiny antennas deployed over a large area. By treating an LIS as a radio image of the environment, we develop sensing techniques that leverage the usage of computer vision combined with machine learning. We test these methods for a scenario where we need to detect whether an industrial robot deviates from a predefined route. The results show that the LIS-based sensing offers high precision and has a high application potential in indoor industrial environments.
\end{abstract}

\section{Introduction}

Massive multiple-input multiple-output (MIMO) is a fundamental technology in the 5 th generation of wireless networks $(5 \mathrm{G})$, with the addition of a large number of antennas per base station as its key feature 11. Looking towards post-5G, researchers are defining a new generation of base stations that are equipped with an even larger number of antennas, giving raise to the concept of large intelligent surface (LIS). Formally, an LIS designates a large continuous electromagnetic surface able to transmit and receive radio waves [2, which can be easily integrated into the propagation environment, e.g., placed on walls. In practice, an LIS is composed of a collection of closely spaced tiny antenna elements. Whilst

* This project has received funding from the European Union's Horizon 2020 research and innovation programme under the Marie Sklodowska-Curie grant agreement No 813999. This work has been submitted to Springer for possible publication. Copyright may be transferred without notice, after which this version may no longer be accessible. 
the performance of LIS in communications has received considerably attention recently $[2] 5]$, the potential of these devices could go beyond communications applications, e.g., environment sensing. Indeed, such large surfaces contain many antennas that can be used as sensors of the environment based on the channel state information (CSI).

Sensing strategies based on electromagnetic signals have been thoroughly addressed in the literature in different ways, and applied to a wide range of applications. For instance, in [6], a real-time fall detection system is proposed through the analysis of the communication signals produced by active users, whilst the authors in 7 use Doppler shifts for gesture recognition. Radar-like sensing solutions are also available for user tracking 8 and real-time breath monitoring [9, as well as sensing methods based on radio tomographic images 10,11. Interestingly, whilst some of these techniques resort solely on the amplitude (equivalently, power) of the receive signals 8, 11, in those cases where sensing small scale variations is needed, the full CSI (i.e., amplitude and phase of the impinging signals) is required 9.10 .

On a related note, machine learning (ML) based approaches are gaining popularity in the context of massive MIMO systems, providing suitable solutions to optimization problems 12 15. Due to the even larger dimensions of the system in extra-large arrays, deep learning may play a key role in exploiting complex patterns of information dependency between the transmitted signals.

The popularization of LIS as a natural next step from massive MIMO gives rise to larger arrays and more degrees of freedom, providing huge amounts of data which can feed ML algorithms. Hence, deep learning arises as a potential solution to exploit the performance of LIS.

In this work, we aim to pave the way to the combined use of both deep learning algorithms and the aforementioned large surfaces, exploring, for first time in the literature, the potential of such a joint solution to sense the propagation environment. Specifically, the contribution of this work is twofold:

- We propose an image-based sensing technique based on the received signal power at each antenna element of an LIS. These power samples are processed to generate a high resolution image of the propagation environment that can be used to feed computer vision algorithms to sense large-scale events.

- A computer vision algorithm, based on transfer learning and support vector machine (SVM), is defined to process the radio images generated by the LIS in order to detect anomalies over a predefined robot route.

The performance of the proposed solution is tested in an indoor industrial scenario, where the impact of the array aperture, sampling period and the interantenna distance is thoroughly evaluated. We show that both larger apertures and smaller separations between the LIS elements render higher resolution images, improving the performance of the system. 


\section{Problem formulation}

We consider an industrial scenario where a robot is following a fixed route, and assume that, due to arbitrary reasons, it might deviate from the predefined route and follow an alternative (undesired) trajectory. Hence, our goal is, based on the sensing signal transmitted by the target device, being able to detect whether the robot is following the correct route or not.

In order to perform the anomalous route detection, we assume that an LIS (i.e., a large array of $M$ closely spaced antennas), is placed in the scenario. Therefore, the sensing problem reduces to determine, from the received signal at each of the LIS elements, if the transmission has been made from a point at the desired route, denoted by $\mathbf{p}_{c} \in \mathbb{R}^{3}$, or from an anomalous one, denoted by $\mathbf{p}_{a} \in \mathbb{R}^{3}$. For the sake of simplicity in a real system implementation, and because we are interested in sensing large scale variations, we resort to the received signal amplitude (equivalently, power). This assumption may lead to simpler system implementations, avoiding the necessity of performing coherent detection.

A classical approach for the aforementioned problem would be performing a hypothesis test based on the received power signal vector. To that end, consider the received complex signal from either $\mathbf{p}_{c}$ or $\mathbf{p}_{a}$ to be

$$
\mathbf{y}_{k}=\mathbf{h}_{k} x+\mathbf{n}_{k}, \quad k=\{c, a\},
$$

with $x$ the transmitted (sensing) symbol, $\mathbf{h}_{k} \in \mathbb{C}^{M \times 1}$ the channel vector from each point and $\mathbf{n}_{k} \sim \mathcal{C N}_{M}\left(\mathbf{0}, \sigma^{2} \mathbf{I}\right)$ the noise vector. Assume, without loss of generality, that $x=1$. Hence, the received power vector is given by

$$
\mathbf{w}_{k}=\left(\left\|y_{1, k}\right\|^{2}, \ldots,\left\|y_{M, k}\right\|^{2}\right)^{T}
$$

where $y_{i, k}$ for $i=1, \ldots, M$ are the elements of $\mathbf{y}_{k}$. The hypothesis test is therefore formulated as

$$
\frac{f_{\mathbf{w}_{c}}\left(\mathbf{w} \mid \mathbf{p}_{c}\right)}{f_{\mathbf{w}_{a}}\left(\mathbf{w} \mid \mathbf{p}_{a}\right)} \underset{\mathbf{p}_{a}}{\gtrless} \frac{\mathbf{p}_{c}}{P_{c}},
$$

where $f_{\mathbf{w}_{k}}(\cdot)$ for $k=\{c, a\}$ is the joint probability function of the received signal from each point, $\mathbf{w}$ is the observation vector, and $P_{a}$ and $P_{c}$ denote the probability of receiving a signal from $\mathbf{p}_{a}$ and $\mathbf{p}_{c}$, respectively. To obtain an optimal estimator, we would need to characterize the joint distribution of the received vector over all the possible anomolaous points, which implies knowing all the possible states of the channels for each path. Also, even in the most simple case, i.e., assuming a pure line-of-sight (LoS) propagation, we would still be unable to distinguish if the two points are in different trajectories or at distinct positions of the same route. Moreover, the a priori probabilities $P_{a}$ and $P_{c}$ are needed, which is a non-trivial task.

In a realistic environment, the complexity of the propagation paths is considerable, and the theoretical analysis becomes cumbersome and site-dependent. Hence, in order to gain insight into how the propagation paths between different positions translate into differences in the received signals, we have to resort on 


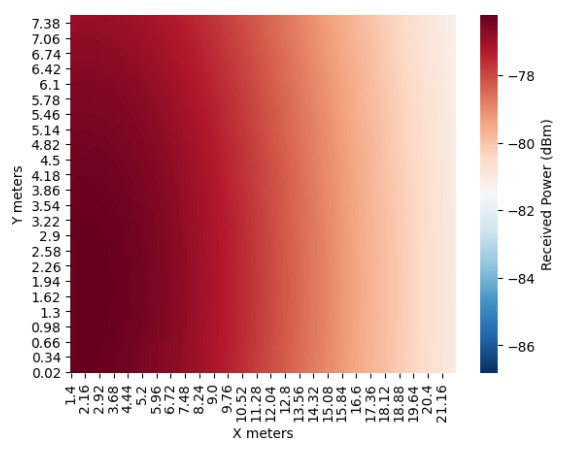

(a) LoS, noiseless.

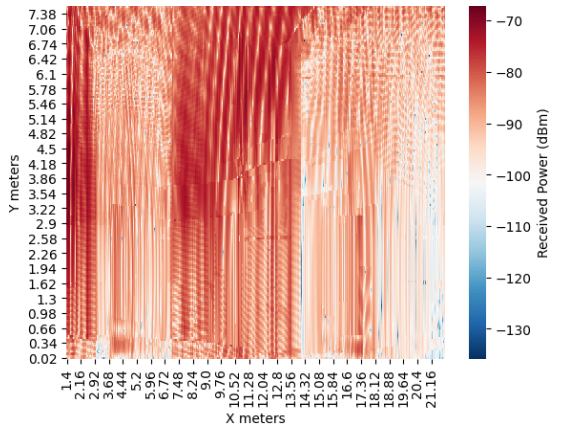

(b) Real scenario, noiseless.

Fig. 1. Holographic images for LOS and Industry scenarios.

machine learning algorithms. This, together with the use of LIS, can provide the necessary information about the propagation environment in order to perform the anomalous route detection.

\section{$3 \quad$ Holographic sensing}

A hologram is a recorded interference pattern as a result of constructive and destructive combinations of the superimposed light-wavefronts, i.e., a photographic recording of a light field [16]. In a wireless context, an LIS could be described as a structure which uses electromagnetic signals impinging in a determined scatterer in order to obtain a profile of the environment. That is, we can use the signal power received at each of the multiple elements of the LIS to obtain a high resolution image of the propagation environment. Using this approach, the complexity of the multipath propagation is reduced to using information represented as an image. This provides a twofold benefit: $i$ ) the massive number of elements that composed the LIS leads to an accurate environment sensing (i.e. high resolution image), and ii) it allows the use of computer vision algorithms and image processing techniques to deal with the resulting images.

As an illustrative example, Fig. 11 shows the holographic images obtained from different propagation environments ( $x$ and $y$ correspond to the physical dimension of the LIS). Specifically, Figs. 1a correspond to a LoS propagation (no scatterers), whilst Fig. $1 \mathrm{~b}$ is obtained from an industrial scenario with a rich scattering. Note that, in the case in which different scatterers are placed, their position and shapes are captured by the LIS and represented in the image. To the best of the authors' knowledge, this is the first time that imaged-based sensing is proposed in the literature. 


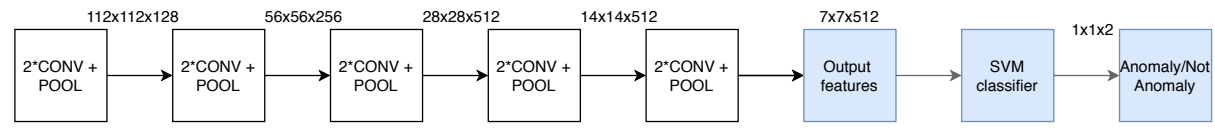

Fig. 2. Proposed model. White and blue blocks refer to VGG19 re-used original architecture and to the additional blocks for our task, respectively.

\section{Machine learning for holographic sensing}

\subsection{Model description}

We here propose the use of a machine learning model to perform the anomalous route classification task, based on the holographic images obtained at the LIS. In our considered problem, the training data is obtained by sampling the received power at certain temporal instants while the target device is moving along the route. In order to reduce both training time and scanning periods, we resort on transfer learning 17]. Thus, a small dataset can be used, improving the flexibility of the system in real deployments. Among the available strategies for this matter, we will use feature representation.

One of the main requirements for transfer learning is the presence of models that perform well on already defined tasks. These models are usually shared in the form of a large number of parameters/weights the model achieved while being trained to a stable state 18 . The famous deep learning Python library, Keras [19], provides an easy way to reuse some of these popular models. We propose the use of a SVM binary classifier, which has been proved to perform correctly when using a large number of features [20]. In our case, we choose the VGG19 architecture 21].

The model is detailed in Fig. 2. In order to perform the feature extraction, we remove the last fully connected layer (FC) that performs the classification for the purpose of VGG19 and modify it for our specific classification task (anomaly/not anomaly in robot's route). We note that the architecture has been frozen for our case, i.e., the weights and biases in VGG19 are fixed and re-used to generate the features to feed the SVM classifier while the regularization parameter $C$ is tuned to prevent overfitting along the training process.

\subsection{Dataset format}

The dataset is obtained by sampling the received signal power at each element of the LIS while the robot moves along the trajectories. Formally, we can define the trajectories as the set of points in the space $\mathbf{P}_{t} \in \mathbb{R}^{N_{p} \times 3}$ being $N_{p}$ the total number of points in the route. Let assume the system is able to obtain $N_{s}$ samples at each channel coherence interval $\forall \mathbf{p}_{j} \in \mathbf{P}_{t}$, being $\mathbf{p}_{j}$ for $j=1, \ldots, N_{p}$ an arbitrary point of the route. Hence, the dataset is conformed by $T=N_{p} \times N_{s}$ samples (monocromatic holographic image snapshots of received power). Each sample is a gray-scale image which is obtained by mapping the received power 
into the range of $[0,255]$. To that end, we apply min-max feature scaling, in which the value of each pixel $m_{i, j}$ for $i=1, \ldots, M$ and $j=1, \ldots, N_{p}$ is obtained as

$$
m_{i, j}=\left\lceil m_{\mathrm{MIN}}+\frac{\left(w_{i, j}-w_{\mathrm{MIN}, j}\right)\left(m_{\mathrm{MAX}}-m_{\mathrm{MIN}}\right)}{w_{\mathrm{MAX}, j}-w_{\mathrm{MIN}, j}}\right\rceil,
$$

where $w_{i, j}$ are the elements of $\mathbf{w}_{j}$ in $(2)$, i.e. $w_{i, j}=\left\|h_{i, j}+n_{i, j}\right\|^{2}, m_{\mathrm{MAx}}=255$ and $m_{\mathrm{MIN}}=0$, and

$$
w_{\mathrm{MAX}, j}=\max _{\{i=1, \ldots, M\}} \mathbf{w}_{i, j}, \quad w_{\mathrm{MIN}, j}=\min _{\{i=1, \ldots, M\}} \mathbf{w}_{i, j}
$$

are the maximum and minimum received power value from a point $\mathbf{p}_{j}$ along the surface.

The input structure supported by VGG19 is a RGB image of $n_{c}=3$ channels. Due to our monocromatic measurements, our original gray-scale input structure is a one-channel image. To solve this problem, we expand the values by copying them into a $n_{c}=3$ channels input structure.

Once the feature extraction is performed, the output is $n_{c}=512$ channels of size $n_{w}=7$ and $n_{h}=7$ pixels. Since SVM works with vectors, the data is reshaped into an input feature vector formed by $7 \times 7 \times 512=25088$ features, meaning our dataset is $\left\{x^{(i)}, y^{(i)}\right\}_{i=1}^{T}$, where $x^{(i)}$ is the $i$-th $n$-dimensional training input features vector (being $n=25088), x_{j}^{(i)}$ is the value of the $j$-th feature, and $y^{(i)}$ is the corresponding desired output label vector.

\section{Model validation}

In order to validate the proposed method, we carried out an extensive set of simulations to analyze the performance of the system. To properly obtain the received power values, we use a ray tracing software, therefore capturing the effects of the multipath propagation in a reliable way. Specifically, we consider Altair Feko Winprop 22.

\subsection{Simulated scenario}

The baseline set-up is described in Fig. 3a, a small size industrial scenario of size $484 \mathrm{~m}^{2}$. We address the detection of the deviation of the target robot (highlighted in red color) when following a fixed route parallel to the bottom wall, in which the LIS is deployed. The distance between the LIS and the desired trajectory is 13.9 $\mathrm{m}$. For the anomalous routes, a separation of $50 / 10 \mathrm{~cm}$ have been simulated to analyze the performance of the system when $\Delta d>>\lambda$ and $\Delta d \approx \lambda$ respectively, as detailed in Fig. 3b.

For these routes, we simulate in the ray tracing software $N_{p}$ points, which corresponds to different positions of the robot in both the correct and anomalous routes. Then, $N_{s}$ holographic image snapshots of the measurements are taken at every $\mathbf{p}_{j}, j=1, \ldots, N_{p}$. The most relevant parameters used for simulation are summarized in Table 1 . 


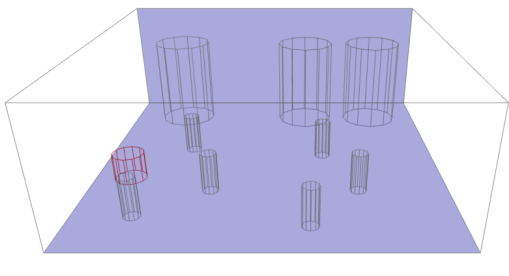

(a) Use case scenario.

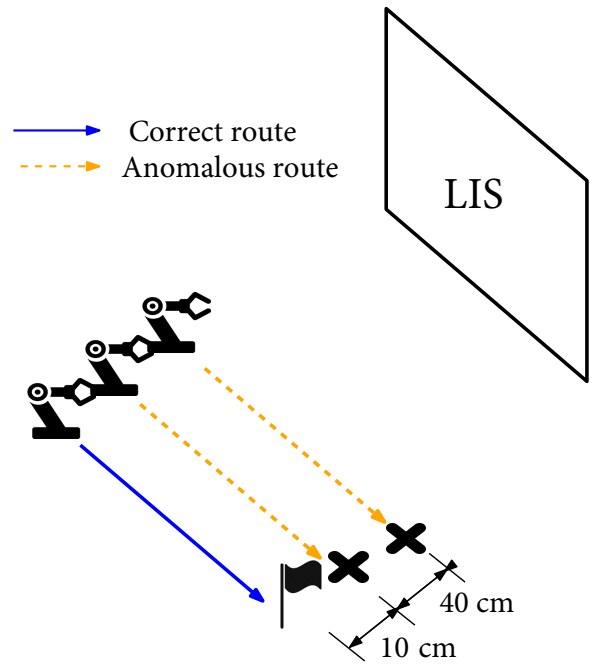

(b) Correct robot route (blue) vs anomalous routes (orange).

Fig. 3. Simulated scenario.

Table 1. Parameters

\begin{tabular}{|c|c|c|c|c|c|}
\hline $\begin{array}{c}\text { Frequency } \\
(\mathrm{GHz})\end{array}$ & $\begin{array}{c}\text { Tx } \\
\text { Power } \\
(\mathrm{dBm})\end{array}$ & $\begin{array}{c}\text { Nray } \\
\text { paths }\end{array}$ & $\begin{array}{c}\text { Antenna } \\
\text { type }\end{array}$ & $\begin{array}{c}\text { Antenna } \\
\text { Spacing }(\mathrm{cm})\end{array}$ & $\begin{array}{c}\text { Propagation } \\
\text { model }\end{array}$ \\
\hline 3.5 & 20 & 20 & Omni & $\frac{\lambda}{2} / \lambda / 2 \lambda$ & Free Space \\
\hline
\end{tabular}

In our simulations, we set $N_{p}=367$ and $N_{s}=10$, thus the dataset is composed of $T=N_{p} \times N_{s}=3670$ radio propagation snapshots containing images of both anomalous and non-anomalous situations, as described in Section 4.2. The dataset is split into a $80 \%$ training set and $20 \%$ for the test set. During the training phase, the obtained optimum regularization value is $C=0.001$, which was identified by using a 5-fold cross-validation strategy [23].

\subsection{Received power and noise modeling}

The complex electric field arriving at the $i$-th antenna element at sample time $t, \widetilde{E}_{i}(t)$, can be regarded as the superposition of each path, i.e 3 ,

$$
\widetilde{E}_{i}(t)=\sum_{n=1}^{N_{r}} \widetilde{E}_{i, n}(t)=\sum_{n=1}^{N_{r}} E_{i, n}(t) e^{j \phi_{i, n}(t)},
$$

\footnotetext{
${ }^{3}$ Note that the electric field also depends on the point $\mathbf{p}_{j}$. However, for the sake of clarity, we drop the subindex $j$ throughout the following subsections.
} 
where $N_{r}$ is the number of paths and $\widetilde{E}_{i, n}(t)$ is the complex electric field at $i$-th antenna from $n$-th path, with amplitude $E_{i, n}(t)$ and phase $\phi_{i, n}(t)$. From (6), and assuming isotropic antennas, the complex signal at the output of the $i$-th element is therefore given by

$$
y_{i}(t)=\sqrt{\frac{\lambda^{2} Z_{i}}{4 \pi Z_{0}}} \widetilde{E}_{i}(t)+n_{i}(t),
$$

with $\lambda$ the wavelength, $Z_{0}=120 \pi$ the free space impedance, $Z_{i}$ the antenna impedance, and $n_{i}(t)$ is complex Gaussian noise with zero mean and variance $\sigma^{2}$. Note that (7) is exactly the same model than (1); the only difference is that we are explicitly denoting the dependence on the sampling instant $t$. For simplicity, we consider $Z_{i}=1 \forall i$. Thus, the power $w_{i}(t)=\left\|y_{i}(t)\right\|^{2}$ is used at each temporal instant $t$ to generate the holographic image, as pointed out before. Finally, in order to test the system performance under distinct noise conditions, the average signal-to-noise ratio (SNR) over the whole route, $\bar{\gamma}$, is defined as $4^{4}$

$$
\bar{\gamma} \triangleq \frac{\lambda^{2}}{4 \pi Z_{0} M T \sigma^{2}} \sum_{t=1}^{T} \sum_{i=1}^{M}\left|\widetilde{E}_{i}(t)\right|^{2},
$$

where $M$ denotes the number of antenna elements in the LIS.

\subsection{Noise averaging strategy}

Noise is critical in image classification performance 24. Normally, in the image processing literature, noise removal techniques assume additive noise in the images [25], which is not the case in our system.

Referring to (1) and (7), since we are considering only received powers, the signal at the output of the $i$-th antenna detector is given by

$$
w_{i}=\left\|\sqrt{\frac{\lambda^{2} Z_{i}}{4 \pi Z_{0}}} \widetilde{E}_{i}+n_{i}\right\|^{2},
$$

where we have dropped the dependence on $t$. Also, let assume the system is able to obtain $S$ extra samples at each channel coherence interval $\forall \mathbf{p}_{j} \in \mathbf{P}$. That is, at each point $\mathbf{p}_{j}$, the system is able to get $N_{s}^{\prime}=N_{s} \times S$ samples. Since the algorithm only expects $N_{s}$ samples from each point, we can use the extra samples to reduce the noise variance at each pixel. To that end, the value of each pixel $m_{i, j}$ is not computed using directly $w_{i, j}$ as in 4 but instead

$$
w_{i, j}^{\prime}=\frac{1}{S} \sum_{s=1}^{S} w_{i, j, s},
$$

\footnotetext{
${ }^{4}$ This is equivalent to average over all the points $\mathbf{p}_{j}$ of the trajectory $\mathbf{P}$.
} 
where $w_{i, j, s}$ denote the received signal power at each extra sample $s=1, \ldots, S$. Note that, if $S \rightarrow \infty$, then

$$
\left.w_{i, j}^{\prime}\right|_{S \rightarrow \infty}=\mathbb{E}\left[w_{i, j} \mid h_{i, j}\right]=\left\|h_{i, j}\right\|^{2}+\sigma^{2},
$$

meaning that the noise variance at the resulting image has vanished, i.e., the received power at each antenna (conditioned on the channel) is no longer a random variable. Observe that the image preserves the pattern with the only addition of an additive constant factor $\sigma^{2}$. This effect is only possible if the system would be able to obtain a very large number $S$ of samples within each channel coherence interval.

\subsection{Performance metrics}

To evaluate the prediction effectiveness of our proposed method, we resort on common performance metrics that are widely used in the related literature. Concretely, we are focusing on the F1-Score which is a metric based on the Precision and Recall metrics 26 and is described as:

- Positive F1-Score $\left(P F_{1}\right)$ and Negative F1-Score $\left(N F_{1}\right)$ as the harmonic mean of precision and recall:

$$
P F_{1}=2 \cdot \frac{\mathrm{PP} \cdot \mathrm{RP}}{\mathrm{PP}+\mathrm{RP}}, \quad \quad N F_{1}=2 \cdot \frac{\mathrm{PN} \cdot \mathrm{RN}}{\mathrm{PN}+\mathrm{RN}} .
$$

Where PP and RP stand for Precision and Recall of the positive class (anomaly) while PN and RN stand for Precision and Recall of the negative class (not anomalous situation).

\section{Numerical results and Discussion}

Generally, in the considered industrial setup, it would be more desirable to avoid undetected anomalies (which may indicate some error in the robot or some external issue in the predefined trajectory) than obtaining a false positive. Hence, all the figures in this section shows the algorithm performance in terms of the $P F_{1}$ metric.

\subsection{Impact of sampling and noise averaging}

To evaluate the impact of both sampling and noise averaging, we consider an LIS compounded by $M=128 \times 128$ antennas and a spacing $\Delta s=\lambda / 2$ for the $\Delta d=50 \mathrm{~cm}$ anomalous route.

For our particular case, $N_{s}^{\prime} \in\{1000,500,100\}$. Then $\forall \mathbf{p}_{j}$ we use $S=\frac{N_{s}^{\prime}}{N_{s}}$ samples for obtaining $N_{s} S$-averaged samples for training the algorithm, being still $T=N_{p} \times N_{s}=3670$. Note that the number of samples $N_{s}^{\prime}$ would depend on the sampling frequency and the second order characterization of the channel, i.e., the channel coherence time and its autocorrelation function. 


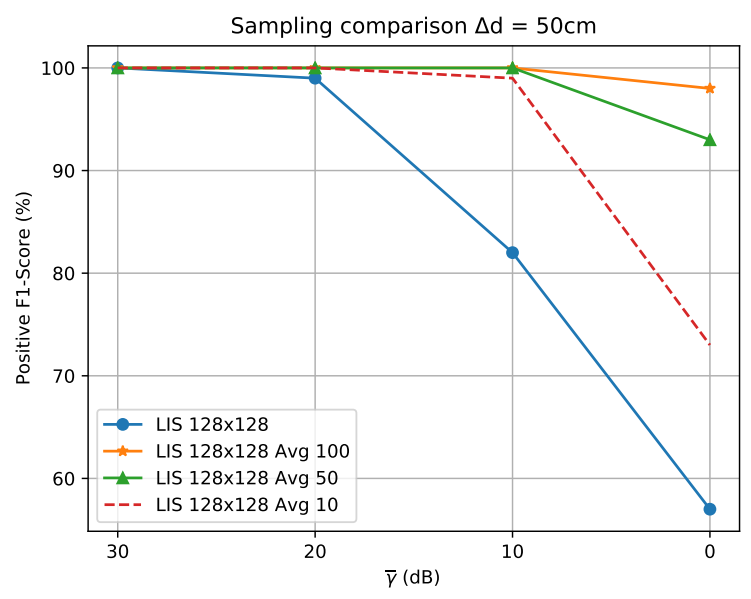

Fig. 4. $P F_{1}$ score averaged noise vs non-averaged.

Figure 4 shows the performance of the system when using non-averaged samples and averaged ones respectively. The blue line represents the system when non-averaged data is being used. When the noise contribution is non-negligible in the interval $\bar{\gamma} \in[10 \mathrm{~dB}, 0 \mathrm{~dB}]$, the detection performance presents a significant drop. Thanks to the averaging, results are significantly improved, even in the critical interval. As expected, when noise level is higher, more samples are needed to preserve the pattern by averaging, being $N_{s}^{\prime}=1000$ the one which yields a better performance. For the following discussions, this sampling strategy will be used, meaning we are using $S=100$ extra samples.

\subsection{Impact of antenna spacing}

To evaluate the impact of inter-antenna distance, we fix the aperture to $5.44 \times$ $5.44 \mathrm{~m}$, we assess the performance in both $\Delta d=50 / 10 \mathrm{~cm}$, and we analyze different spacings with respect to the wavelength $(\lambda / 2, \lambda$ and $2 \lambda)$.

The performance results for the distinct configurations are depicted in Fig. 5. As observed, the spacing of $2 \lambda$ - which is far from the concept of LIS is presenting really inaccurate results showing that the spatial resolution is not enough. We can conclude that the quick variations along the surface provide important information to the classifier performance. Besides, this information becomes more important the lower the distance between the routes is. The performance drop due to the closer distances among the routes is related to the pattern classification. The closer the routes are, the more similar the pattern is making more challenging to perform the detection. However, reducing the antenna spacing even more can improve the performance when routes are even closer. What is more, the effect of antenna densification for a given aperture is highlighted and it can be seen that the lowest spacing leads to the best results. 


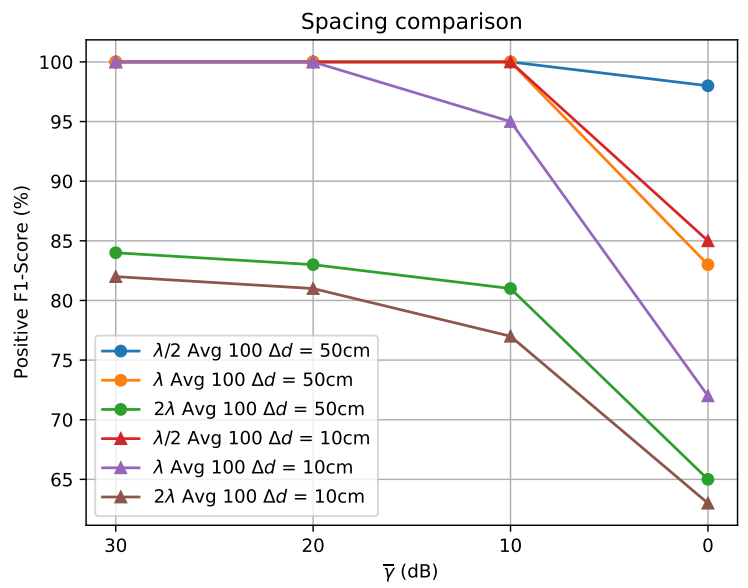

Fig. 5. $P F_{1}$ score antenna spacing

\subsection{LIS aperture comparisons}

In this case LIS with different apertures have been evaluated. The spacing is fixed to $\lambda / 2$.

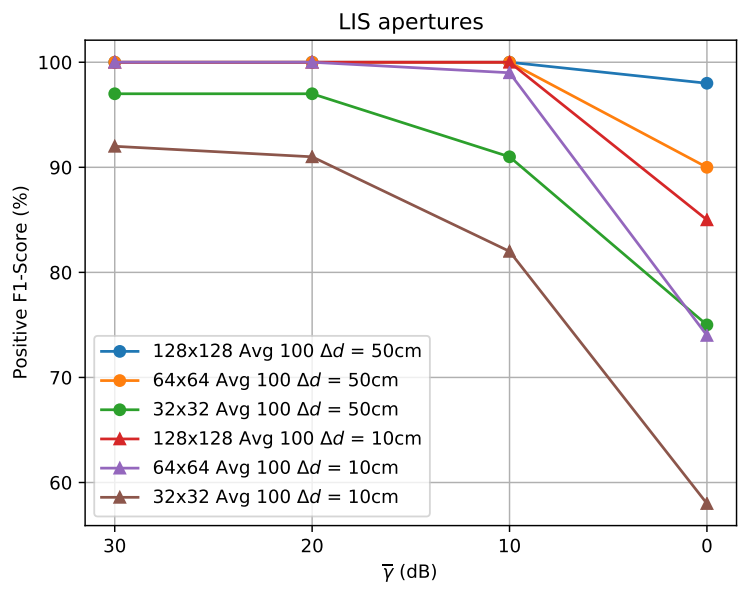

Fig. 6. Different LISs apertures comparison

Looking at Fig. 6, the aperture plays a vital role in the sensing performance. Increasing the number of antennas leads to a higher resolution image, being able 
to capture the large-scale events occurring in the environment more accurately. Note the usage of incoherent detectors is yielding to a good performance when the aperture is large enough. The key feature for this phenomena is the LIS pattern spatial consistency, i.e., the ability of representing the environment as a continuous measurement image.

\section{Conclusions}

We have shown the potential of LIS for sensing the environment, being able to provide high resolution radio images of the propagation environment that can be processed by existing and versatile solutions in the context of computer vision algorithms. This sensing technique, which we consider appropriate to refer to as holographic sensing, arises as a robust solution to capture the large scale events of a target scenario, with the inherent advantage that the received signal phase does not need to be estimated. The combined usage of both LIS and machine learning algorithms may be potentially used in the context of cognitive radio and multiuser massive MIMO as a support technology to enhance the performance of these systems.

\section{References}

1. Jeffrey G Andrews, Stefano Buzzi, Wan Choi, Stephen V Hanly, Angel Lozano, Anthony CK Soong, and Jianzhong Charlie Zhang. What will 5G be? IEEE J. Sel. Areas Commun., 32(6):1065-1082, 2014.

2. S. Hu, F. Rusek, and O. Edfors. Beyond massive MIMO: The potential of data transmission with large intelligent surfaces. IEEE Trans. Signal Process, 66(10):2746-2758, 2018.

3. Ertugrul Basar. Transmission through large intelligent surfaces: A new frontier in wireless communications. In EuCNC: Eur. Conf. Netw. Commun., pages 112-117. IEEE, 2019.

4. Davide Dardari. Communicating with large intelligent surfaces: Fundamental limits and models, 2019.

5. Emil Björnson and Luca Sanguinetti. Power scaling laws and near-field behaviors of massive mimo and intelligent reflecting surfaces, 2020.

6. Hao Wang, Daqing Zhang, Yasha Wang, Junyi Ma, Yuxiang Wang, and Shengjie Li. Rt-fall: A real-time and contactless fall detection system with commodity WiFi devices. IEEE Trans. Mobile Comput., 16(2):511-526, 2016.

7. Qifan Pu, Sidhant Gupta, Shyamnath Gollakota, and Shwetak Patel. Whole-home gesture recognition using wireless signals. In Proc. 19th Annual Inter. Conf. Mobile Comput. \& Netw., pages 27-38, 2013.

8. Yang Zhao, Neal Patwari, Jeff M Phillips, and Suresh Venkatasubramanian. Radio tomographic imaging and tracking of stationary and moving people via kernel distance. In 2013 ACM/IEEE Inter. Conf. Inf. Process. Sensor Networks (IPSN), pages 229-240. IEEE, 2013.

9. Fadel Adib, Zachary Kabelac, Hongzi Mao, Dina Katabi, and Robert C Miller. Real-time breath monitoring using wireless signals. In Proc. 20th Annual Inter. Conf. Mobile Comput. Netw., pages 261-262, 2014. 
10. Mingmin Zhao, Tianhong Li, Mohammad Abu Alsheikh, Yonglong Tian, Hang Zhao, Antonio Torralba, and Dina Katabi. Through-wall human pose estimation using radio signals. In Proc. IEEE Conf. Comput. Vis. Pattern Recognit., pages 7356-7365, 2018.

11. Joey Wilson and Neal Patwari. Radio tomographic imaging with wireless networks. IEEE Trans. Mobile Comput., 9(5):621-632, 2010.

12. Jingon Joung. Machine learning-based antenna selection in wireless communications. IEEE Commun. Lett., 20(11):2241-2244, 2016.

13. O. T. Demir and E. Bjornson. Channel estimation in massive MIMO under hardware non-linearities: Bayesian methods versus deep learning. IEEE O. J. Commun. Soc., 1:109-124, 2020.

14. X. Ma and Z. Gao. Data-driven deep learning to design pilot and channel estimator for massive mimo. IEEE Trans. Veh. Technol., 69(5):5677-5682, 2020.

15. H. Huang, J. Yang, H. Huang, Y. Song, and G. Gui. Deep learning for superresolution channel estimation and doa estimation based massive mimo system. IEEE Trans. Veh. Technol., 67(9):8549-8560, 2018.

16. RRA Syms. Practical volume holography clarendon. Oxford, 19902:125, 1990.

17. Sinno Jialin Pan and Qiang Yang. A survey on transfer learning. IEEE Trans. Knowl. Data Eng., 22(10):1345-1359, 2009.

18. Dipanjan Sarkar, Raghav Bali, and Tamoghna Ghosh. Hands-On Transfer Learning with Python: Implement advanced deep learning and neural network models using TensorFlow and Keras. Packt Publishing Ltd, 2018.

19. François Chollet et al. Keras. https://keras.io, 2015.

20. Christopher M Bishop. Pattern recognition and machine learning. Springer, 2006.

21. Karen Simonyan and Andrew Zisserman. Very deep convolutional networks for large-scale image recognition. arXiv preprint arXiv:1409.1556, 2014.

22. Winprop, altair engineering, inc. https//www.altairhyperworks.com/winprop

23. Davide Anguita, Alessandro Ghio, Sandro Ridella, and Dario Sterpi. K-fold cross validation for error rate estimate in support vector machines. In DMIN, pages 291-297, 2009.

24. Prasun Roy, Subhankar Ghosh, Saumik Bhattacharya, and Umapada Pal. Effects of degradations on deep neural network architectures. arXiv preprint arXiv:180\%.10108, 2018.

25. Thomas B Moeslund. Introduction to video and image processing: Building real systems and applications. Springer Science \& Business Media, 2012.

26. David Martin Powers. Recall \& precision versus the bookmaker. International Conference on Cognitive Science, 2003. 